\title{
Intelligent Lossy Compression Method of Providing a Desired Visual Quality for Images of Different Complexity
}

\author{
Fangfang LI ${ }^{\mathrm{a}, \mathrm{b}}$, Vladimir Vasilyevich LUKIN ${ }^{\mathrm{b}, 1}, \mathrm{Krzysztof}_{\mathrm{OKARMA}}^{\mathrm{c}}$, Yanjun FU ${ }^{\mathrm{a}}$ \\ and Jiangang DUAN ${ }^{\mathrm{d}}$ \\ ${ }^{a}$ Key Laboratory of Nondestructive Testing, Education Ministry of China, Nanchang \\ Hangkong University, Fenghe South Ave.696, Nanchang 330063, China \\ ${ }^{\mathrm{b}}$ Dept of Information-Communication Technologies, National Aerospace University - \\ KhAI, Chkalova Str.17, Kharkov 61070, Ukraine \\ ${ }^{\mathrm{c}}$ Department of Signal Processing and Multimedia Engineering, West Pomeranian \\ University of Technology in Szczecin, Szczecin 70-313, Poland \\ d AECC Shenyang Liming Aero-Engine Co., LTD., Shenyang 110043, China
}

\begin{abstract}
Lossy compression plays a vital role in modern digital image processing for producing a high compression ratio. However, distortion is unavoidable, which affects further image processing and must be handled with care. Providing a desired visual quality is an efficient approach for reaching a trade-off between introduced distortions and compression ratio; it aims to control the visual quality of the decompressed images and make them not worse than the required by a user. This paper proposes an intelligent lossy compression method of providing a desired visual quality, which considers the complexity of various images. This characteristic is utilized to choose an appropriate average rate-distortion curve for an image to be compressed. Experiments have been conducted for Discrete Cosine Transform (DCT) based lossy compression coder, Peak Signal-Noise Ratio (PSNR) has been employed to evaluate the visual quality. The results show that our new method has the ability to provide a general improvement of accuracy, and the proposed algorithm for classifying image complexity by entropy calculation is simpler and faster than earlier proposed counterparts. In addition, it is possible to find "strange" images which produce the largest errors in providing a desired quality of compression.
\end{abstract}

Keywords. Lossy compression, desired visual quality, PSNR, complexity, entropy.

\section{Introduction}

Nowadays, image size has the trend to increase dramatically with the development of imaging technology and the widespread use of digital images [1-3]. Massive large size images result in a high cost of storage resources and low time efficiency of transferring via communication lines. Therefore, compression plays an important role in image processing when it is required to reduce the data size and becomes necessary before

1 Corresponding Author, Vladimir Vasilyevich LUKIN, Dept of Information-Communication Technologies, National Aerospace University, Chkalova Str.17, Kharkov 61070, Ukraine; E-mail: lukin@ai.kharkov.com. 
storage and transmission. Generally, the compression techniques are divided into two categories, namely lossless and lossy. Lossless compression is able to produce an ideally reconstructed image with a limited compression ratio (CR) $[4,5]$. In contrast, lossy compression outperforms the lossless compression in terms of $\mathrm{CR}$, but the distortions are unavoidably introduced [4, 6, 7]. Lossy compression has been widely used in applications where a certain degree of losses is acceptable due to its higher CR, and a great number of coders has been proposed, most of them focus on increasing the CR with limited degradation of compressed image quality [4, 8-10].

A task that appears is to provide the desired quality quickly enough and accurately (quality of compressed images can differ a lot for a given quantization step or bpp of a used coder). One way out is to apply an iterative procedure that performs multiple compression/decompression, image quality estimation, and coder parameter changing $[11,12]$. However, such a procedure might require a considerable and a priori unpredictable number of iterations and, thus, take a lot of time. An alternative is to apply two-step procedures[13-17] that employ average rate-distortion curves obtained in advance to determine the coder parameter at the first step, one compression/decompression to determine quality for a particular image to be compressed, coder parameter correction, and the second (final) step compression. The drawback of this procedure is that sometimes its accuracy in providing compressed image quality can be insufficient $[15,17]$. This happens if the characteristics of a particular image differ a lot from "average" ones. Then, it is possible to carry out a simple classification of images before their lossy compression[14] and use different average curves for different groups of images. This partly helps, but there can be "strange" images. Besides, it is desired to carry out preliminary classification faster and more reliably.

In this paper, we show that image classification can be done on the basis of entropy calculation and comparison to a set of thresholds. This allows fast detection of "strange" images and division of other images into three groups (simple, middle, and high complexity). This results in better (more accurate) providing of the desired PSNR in the range where introduced distortions are visible.

\section{Method}

Generally, some parameters in lossy compression are used to control the CR and, simultaneously, visual quality of compressed image, whether this coder is DCT-based, or DWT-based or other. In the DCT-based coder AGU [18] considered in this paper, the parameter is quantization step (QS). In previous works $[13,15]$, we have studied the dependence of visual quality on QS, and several visual metrics have been investigated. It has been demonstrated that there are common trends where a larger QS corresponds to lower visual quality for each particular image. However, the metric values for different images vary greatly for a given QS, particularly in PSNR and especially if PSNR is about $30 \mathrm{~dB}$. Therefore, the accuracy of the two-step method on PSNR is lower than other metrics and can be even unsatisfactory.

To improve the accuracy of the two-step method of lossy compression, the complexity of images was introduced in [14]. High texture image often gets the lower PSNR than simple texture image for the same QS, and this means that image complexity is another factor that determines visual quality. Consequently, an alternative approach proposed was to divide images into simple and complex structure ones and have two average rate-distortion curves, respectively. The classification strategy in [14] was either 
to predict the PSNR value of an image set for some QS value(s) or to calculate CR for lossless compression ( $\left.\mathrm{CR}_{\text {lossless }}\right)$. It was supposed that these parameters being compared to thresholds are able to classify images. The calculation of $\mathrm{CR}_{\text {lossless }}$ is time-consuming compared to the two-step method, the PSNR on QS equal to 30 can be utilized to replace it for the relatively high coefficient correlation of both compared to other QS values, they are 0.32 for Pearson and 0.13 for Spearman, but this also requires additional computations. The adaptive two-step method was implemented as follows: the prediction algorithm of PSNR on test image was conducted firstly, then classification is carried out; finally, the suitable average rate-distortion curve is chosen to run the two-step procedure. This approach partly helps to improve the accuracy, but the prediction of PSNR is still not fast as we desired. Another problem is that the extreme images are difficult to point out, which has strange performance for the two-step method.

In this paper, we propose a new approach to solve the problems mentioned above. Our idea is based on the assumption that the entropy is able to characterize the image complexity. In fact, entropy has been used to determine the complexity of images to detect defects on 3D printed surfaces [19]. To verify the theoretical feasibility, the entropy data have been obtained for our image set (including 61 gray-scale images of the size of $512 \times 512$ pixels). The result is shown in figure 1 .

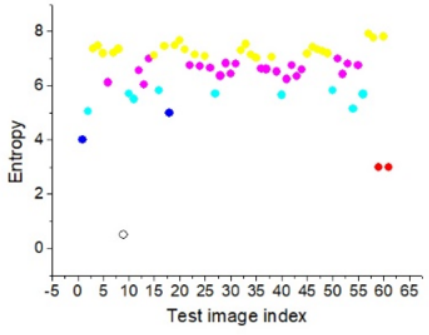

Figure 1. Entropy values for 61 gray-scale images.

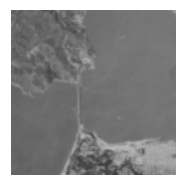

(a)

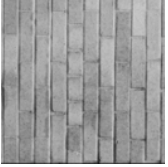

(b)

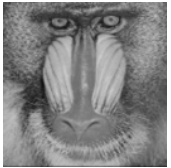

(c)

Figure 2. Image examples, (a) simple image, (b) middle complexity image, (c) complex image.

For 61 images, entropy varies from 0.5 to 7.9 , along with $\mathrm{CR}_{\text {lossless }}$ varying from 100.7 to 1 . The correlation coefficients for these two indicators of image complexity are -0.74 for Pearson, -0.88 for Spearman, and -0.76 for Kendall, respectively. The larger $\mathrm{CR}_{\text {lossless }}$ corresponds to simpler images; meanwhile, the entropy value is smaller, and vice versa. It is easy to roughly divide the images set into three levels according to the entropy of an image: 1) complex image if entropy $>7$;2) middle complexity image if $6<$ entropy $\leq 7$; 3) simple complexity image if entropy $<6$. Three examples are shown in figure 2, corresponding to three levels of complexity.

Figure 2 (a) is the remote sense image Frisco, which has a large uniform area as the background, and it belongs to the simple image set according to entropy (5.8166). Figure 2(b) shows a texture image (numbered as \#12), which has strong texture information, and it belongs to the middle complexity image set since its entropy equals 6.799 . The last one is the image Baboon with the entropy equal to 7.3579 , which is the typical complex image for its rich texture information and complex composition. Based on the comparison of the three images, it proves that our classification strategy roughly corresponds to the complexity of the image.

Similarly to [14], the average rate-distortion curves have been obtained for three level basic image sets, 4 images in the simple image set, 15 images in the middle complexity image set, and 17 images in the complex image set. These curves are shown 
in figure 3; the curves of three images (shown in Figure 2) are also present in the corresponding plots. Three average curves have the trend in common, PSNR decreases monotonically along with QS increasing, and the average curves are approximately "locally parallel" to other curves of the corresponding complexity images, but the curvature deviations of different images sets are different. Among them, the images in the simple image set have the largest diversity compared to the other two sets.

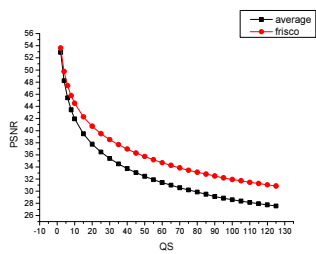

(a)

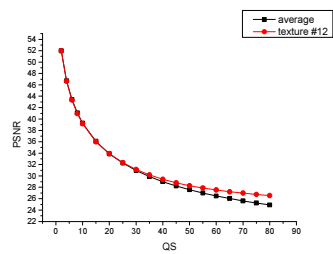

(b)

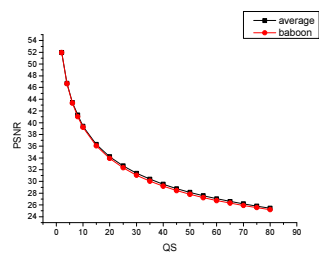

(c)

Figure 3. Dependence of PSNR on QS, (a) simple images; (b) middle complexity images; (c) complex images.

According to the two-step method, these curves are the most important data for calculating QS values for the first and second steps of compression for providing a desired visual quality. First, the estimated QS $\left(\mathrm{QS}_{\text {est }}\right)$ value is obtained from one point on the average rate-distortion curve (chosen according to the entropy of an image be

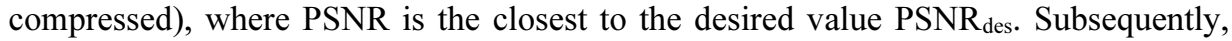
equation (1) is employed to calculate the initial $\mathrm{QS}\left(\mathrm{QS}_{\text {init }}\right)$ used in the first step compression, where $\mathrm{M}^{\prime}$ denotes the derivate of the curve in the point $\mathrm{QS}_{\text {est. }}$.

$$
Q S_{\text {init }}=Q S_{\text {est }}+\frac{P S N R_{\text {des }}-P S N R_{\text {ave }}}{M \prime}
$$

The first step compression is conducted with $\mathrm{QS}_{\text {init, }}$ then decompress and calculate the visual quality of decompressed image with respect to the original image. This initial PSNR ( PSNR $_{\text {init }}$ ) is usually quite close to PSNR des, but it can be not close enough to meet the requirement of a user; therefore, the second step is needed to reduce the error. The QS is corrected by equation (2) using PSNR init. The second step is conducted with the corrected $\mathrm{QS}\left(\mathrm{QS}_{\mathrm{des}}\right)$ and produces a compressed image file, which provides a PSNR $\left(\mathrm{PSNR}_{\mathrm{pro}}\right.$ ) closer to $\mathrm{PSNR}_{\mathrm{des}}$ than $\mathrm{PSNR}_{\text {init }}$.

$$
Q S_{\text {des }}=Q S_{\text {init }}+\frac{P S N R_{d e s}-P S N R_{\text {init }}}{M \prime}
$$

\section{Results and Discussion}

Test experiments were carried out on 61 images (including 36 basic images), and statistical results are shown in table 1 , where three PSNR values $(40,35$, and $30 \mathrm{~dB})$ were selected as the desired quality. We have determined the variance of PSNR init $_{\text {for }}$ the first step $\left(V_{A R}\right)$ and variance of PSNR $R_{\text {pro }}$ for the second step $\left(V_{\text {fir }}\right), \mathrm{RAX}_{\Delta \mathrm{sinal}}$ denotes the maximum absolute error between PSNR pro $_{\text {and }}$ PSNR $_{\text {des }}$ in the data group. The result proves that the second step compression has effectively reduced the error; the variance of PSNR $\mathrm{Pro}_{\text {ro }}$ does not exceed $1.5 \mathrm{~dB}^{2}$. Compared to the basic two-step method, the accuracy has been, in general, improved by our new approach with entropy classification. However, there is still some detailed information that needs to be noted. The errors in the simple image set are larger than in the other two sets, and the largest error in it occurs 
in the desired PSNR equal to 40dB, while in the other two sets, the largest error occurs in the lowest desired PSNR (as it was in our works [13-15]).

The problems for the simple image set are due to three "strange" images presented in figure 4; their dependences of PSNR on QS are given. As one can see, these are artificially created images with a limited number of value levels (specific histograms of image values). Rate-distortion curves for them might not be monotonous or differ a lot from the average curve. This causes problems in using the expressions (1) and (2) in providing the desired quality. Then, preliminary calculation of entropy can show in practice that one deals with a "strange" image (if entropy is smaller than 3, "be careful").

Table 1. Statistical results

\begin{tabular}{ccccc}
\hline Image set & PSNR $_{\text {des }}$ & VAR $_{\text {fis }}$ & VAR $_{\text {sec }}$ & MAX $_{\text {sfinal }}$ \\
\hline \multirow{2}{*}{ Simple test image set } & 40 & 26.1129 & 1.4352 & 4.4539 \\
& 35 & 20.153 & 1.4246 & 2.8279 \\
Medium test image set & 30 & 15.2624 & 1.1545 & 1.9385 \\
& 40 & 0.1583 & 0.0033 & 0.2394 \\
Complex test image set & 35 & 0.6986 & 0.486 & 0.8277 \\
& 30 & 2.4614 & 0.4111 & 1.907 \\
& 40 & 0.3173 & 0.0238 & 0.5398 \\
& 35 & 1.3866 & 0.1221 & 1.4162 \\
\hline
\end{tabular}

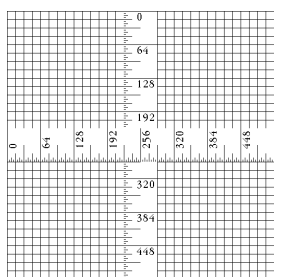

(a)

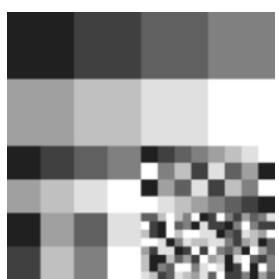

(b)

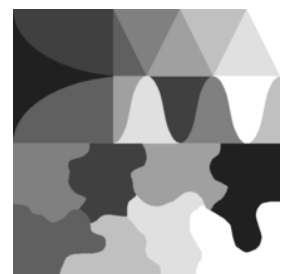

(c)

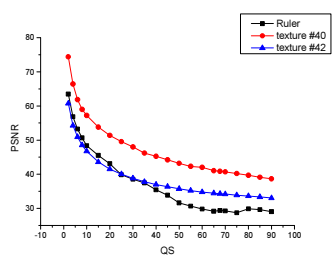

(d)

Figure 4. Special ("strange") images and their dependences of PSNR on QS: (a) Ruler, entropy=0.5; (b) texture $\# 40$, entropy $=2.9994$; (c) texture $\# 42$, entropy $=2.9833$; (d) Rate-distortion curves for these images.

\section{Conclusions}

We have considered providing a desired quality of lossy compressed images (characterized by PSNR) for the DCT-based coder AGU. Principal problems have been demonstrated dealing with the fact that the complexity of an image subject to compression can differ a lot. Due to this, rate-distortion characteristics for different images differ a lot. Following the earlier introduced approach, we have proposed to carry out preliminary classification of image complexity that allows distinguishing the images into simple, medium, and complex (and to detect "strange" images as well). The advantage of the proposed approach to classification is that it is based on entropy that can be calculated quickly and then compared to thresholds. Since several average ratedistortion curves are pre-determined, applying the two-step compression method using pre-classification results is possible. This allows sufficient improvement of accuracy in providing a desired quality of compressed images. 


\section{Acknowledgments}

The research is partially co-financed by the Polish National Agency for Academic Exchange (NAWA) and the Ministry of Education and Science of Ukraine under the project No. PPN/BUA/2019/1/00074 entitled "Methods of intelligent image and video processing based on visual quality metrics for emerging applications".

\section{References}

[1] Kougianos E, Mohanty S P, Coelho G, Albalawi U, and Sundaravadivel P 2016 Design of a highperformance system for secure image communication in the internet of things, IEEE Access, vol. 4, pp. 1222-1242

[2] Chi M, Plaza A, Benediktsson J A, Sun Z, Shen J, and Zhu Y 2016 Big data for remote sensing: Challenges and opportunities, Proceedings of the IEEE, vol. 104, no. 11, pp. 2207-2219

[3] Ma Y et al. 2015 Remote sensing big data computing: Challenges and opportunities, Future Generation Computer Systems, vol. 51, pp. 47-60

[4] Hussain A J, Al-Fayadh A, and Radi N 2018 Image compression techniques: A survey in lossless and lossy algorithms, Neurocomputing, vol. 300, pp. 44-69

[5] Reddy M P 2018 The lossless medical image compression for telemedicine applications with delimiter, Jour of Adv Research in Dynamical Control Systems, vol. 10, no. 3, pp. 74-79

[6] D. Tao, S. Di, X. Liang, Chen Z, and Cappello F,2018, Fixed-PSNR Lossy Compression for Scientific Data, presented at the 2018 IEEE International Conference on Cluster Computing (CLUSTER), Belfast, UK

[7] Penna B, Tillo T, Magli E, and Olmo G 2007 Transform coding techniques for lossy hyperspectral data compression, IEEE Transactions on Geoscience Remote Sensing, vol. 45, no. 5, pp. 1408-1421

[8] Johnston $\mathrm{N}$ et al.,2018, Improved lossy image compression with priming and spatially adaptive bit rates for recurrent networks, in Proceedings of the IEEE Conference on Computer Vision and Pattern Recognition, 2018, pp. 4385-4393

[9] Doss S, Pal S, Akila D, Jeyalaksshmi S, Jabeen T N, and Suseendran G 2020 Satellite image remote sensing for identifying aircraft using SPIHT and NSCT, IEEE Signal processing magazine vol. 7, no. 5, pp. 631-634

[10] Albalawi U, Mohanty S P, and Kougianos E,2015, A hardware architecture for better portable graphics (BPG) compression encoder, in 2015 IEEE International Symposium on Nanoelectronic and Information Systems, 2015, pp. 291-296

[11] Zhang X 2010 Lossy compression and iterative reconstruction for encrypted image, IEEE transactions on information forensics security, vol. 6, no. 1, pp. 53-58

[12] Pandey A, Saini B S, Singh B, and Sood N J M 2020 Quality controlled ECG data compression based on 2D discrete cosine coefficient filtering and iterative JPEG2000 encoding, vol. 152, p. 107252

[13] Li F, Krivenko S, and Lukin V,2020, A Two-step Approach to Providing a Desired Visual Quality in Image Lossy Compression, in 2020 IEEE 15th International Conference on Advanced Trends in Radioelectronics, Telecommunications and Computer Engineering (TCSET), 2020, pp. 502-506

[14] Li F, Krivenko S, and Lukin V,2020, Adaptive two-step procedure of providing desired visual quality of compressed image, in Proceedings of the 2020 4th International Conference on Electronic Information Technology and Computer Engineering, 2020, pp. 407-414

[15] Li F, Krivenko S, and Lukin V 2020 Analysis of two-step approach for compressing texture images with desired quality, Aerospace Technic and Technology, vol. 161, no. 1, pp. 50-58

[16] Li F, Krivenko S, and Lukin V,2020, A Two-step Procedure for Image Lossy Compression by ADCTC With a Desired Quality, in 2020 IEEE 11th International Conference on Dependable Systems, Services and Technologies (DESSERT), 2020, pp. 307-312

[17] Li F, Krivenko S, and Lukin V 2020 Two-step providing of desird quality in lossy image compression by SPIHT, Radio electronic and computer systems, vol. 94, no. 2, pp. 22-32

[18] Ponomarenko N. (2008). AGU download page. Available: http://ponomarenko.info/agu.htm

[19] Okarma K and Fastowicz J 2020 Improved quality assessment of colour surfaces for additive manufacturing based on image entropy, Pattern Analysis Applications, vol. 23, no. 3, pp. 1035-1047 